\title{
An Approach for Using of Poly Glycolic Acid (PGA) in Reference Standard Dosimetry: PGA/ESR Dosimetry System Response Curve and Post Irradiation Stability
}

\author{
Arbi Mejri ${ }^{1,2^{*}}$, Haikel Jelassi ${ }^{2}$, Khaled Farah ${ }^{2}$, Ahmad Hichem Hamzaoui ${ }^{3}$, Hichem Eleuch ${ }^{4}$ \\ ${ }^{1}$ Ionizing Radiation Dosimetry Laboratory, National Center for Nuclear Sciences and Technologies, \\ Tunis, Tunisia \\ ${ }^{2}$ Research Unit: Control and Development of Nuclear Technology for Peaceful Uses, National Center for Nuclear Sciences and \\ Technologies, Tunis, Tunisia \\ ${ }^{3}$ National Institute of Scientific and Technical Research, Tunis, Tunisia \\ ${ }^{4}$ Departments of Physics and Astronomy, Institute for Quantum Science and Engineering, Texas A \& M University, \\ College Station, Texas, USA \\ Email: arbi.mejri@cnstn.rnrt.tn
}

Received February 16, 2012; revised March 23, 2012; accepted April 7, 2012

\begin{abstract}
Reference Standard Dosimeters are used to calibrate radiation environments and routine dosimeters. It can also be used in routine dosimetry applications for radiation processing where higher quality dosimetry measurements are required. Electron Spin Resonance (ESR) is a well-established Reference Standard Dosimetry system in industrial applications of ionising radiation, and its use is also proposed in radiation therapy and accident dosimetry. In the present experimental work, PGA solid state dosimeter (SSD) has been investigated using ESR spectroscopy to study the gamma radiation response of this material and to evaluate its dosimetric characteristics: dose response, room temperature fading, heat treatment effect during post-irradiation storage. Results obtained up to now confirm that PGA seems to be suitable material for ESR dosimetry applications.
\end{abstract}

Keywords: Dosimetry; Solid State Dosimeter (SSD); Poly Glycolic Acid (PGA); Electron Spin Resonance (ESR); Reference Standard Dosimetery; Post-Irradiation Thermal Treatments; Fading Behaviour

\section{Introduction}

The ionizing radiation produces electron-hole pairs which individually become trapped at various defect sites (radicalic species) in the polymer structure. The commonly standard spectroscopic technique used for the characterisation of radicalic species, their stabilities and decay kinetics is Electron Paramagnetic Resonance (EPR), also known as Electron Spin Resonance (ESR).

Semiconductors and polymers are examples of materials that have been studied by ESR spectroscopy through the detection of paramagnetic species [1-3]. It has therefore been the main experimental technique in understanding the effect of ionizing radiation on polymers.

Radiation-induced radicals in certain material may be suitable for some applications in radiation processing dosimetry, it provided that radicals having long-term stability can be used in the establishment of relations between radiation dose and radical concentration.

The more used material in Dosimetric applications for

${ }^{*}$ Corresponding author.
ESR dosimetry is the alanine [4-6] due to its good dosimetric properties, such as sensitivity, stability of the ESR signal with time [6]. Despite the alanine is accepted as reference standard dosimeter by International Atomic Energy Agency (IAEA), its sensitivity is not high for low doses and it has a very high cost. Therefore there are many attempts to characterize a new Reference Standard Dosimeters with better radiation sensitivity to substitute alanine [4,7-11]. The new material should have a stable radical per radiation energy; a sharp linewidth and thermal stability at room temperature.

One of these new materials already studied by X-Band ESR measurements is Poly Glycolic Acid (PGA) used for many years in medical and surgical applications [1215]. The molecular structure of PGA and the free radicals formed by its interaction with radiation are shown in Figure 1. The free radical produced by radiation in PGA which is predominant at room temperature is the molecular species - $\mathrm{CH}^{\circ}$-COO- Radical I [12]. In ESR Spectrum it's traduced by a doublet signal (Figure 2) and it is formed by Hydrogen abstraction from a Methylene 


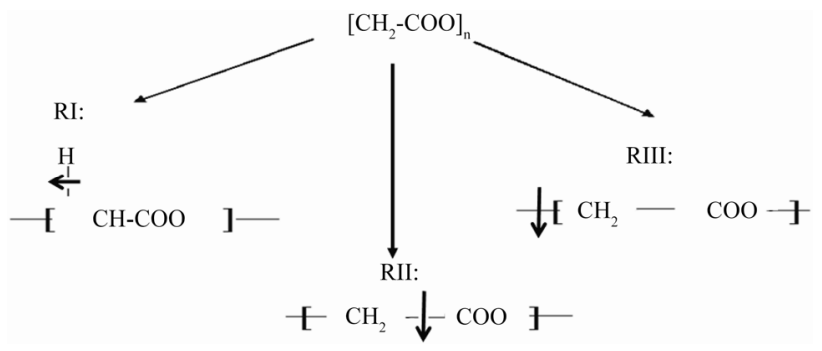

Figure 1. Molecular structure of PGA and the most probable free radicals formed.

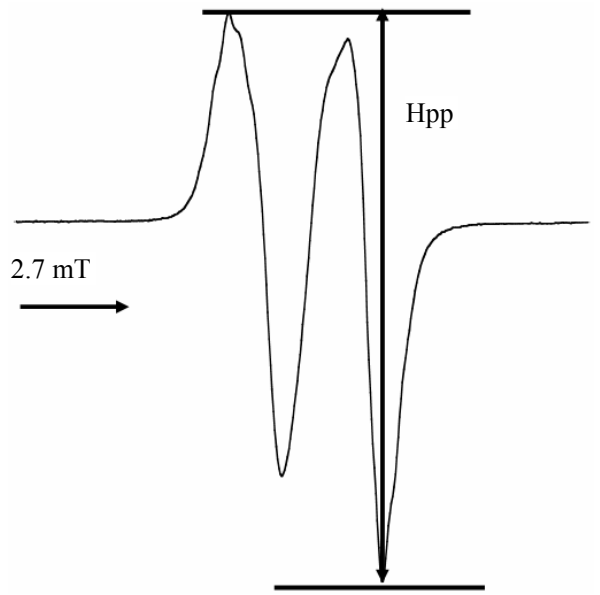

Figure 2. Peak to Peak higher (Hpp) of ESR spectra related to PGA irradiated at a dose of $25 \mathrm{kGy}$.

group located adjacent to Carbonyl group in the polymer backbone [12]. At room Temperature there is essentially no contribution to the spectrum from radicals II and III.

One of the goals of the present work was to analyze the response of PGA dosimeter for possible application as Reference Standard Dosimeter using ESR technique to ensure quality of high and low dose dosimetry applications.

PGA dosimeter has a good sensitivity for low doses, a wide useful dose range and it does not require special preparation. The main difficulty presented by PGA dosimeter is the undesirable strong initial fading. Many authors overcame this problem (case of other dosimetry system) by taking measurements at the most adequate time intervals after irradiation [16,17]. In order to solve this problem in our work, the PGA dosimeters was submitted to different post-irradiation thermal treatments from $60^{\circ} \mathrm{C}$ up to $140^{\circ} \mathrm{C}$ and the effect of these treatments on the fading behaviour of the dosimeters was studied up to
45 days after irradiation at room temperature.

\section{Experimental}

\subsection{Poly Glycolic Acid (PGA) Polymer}

Poly Glycolic Acid was obtained from Boehringer Ingelheim of Germany. The characteristics of the materials, according to the supplier, are given in Table $\mathbf{1}$.

\subsection{Irradiation}

The irradiations have been performed in air at the Tunisian semi-industrial ${ }^{60} \mathrm{Co}$ gamma-irradiation facility [18]. The dose rate was established with the alanine/EPR dosimetry system in term of absorbed dose traceable to the National Physical Laboratory, UK. Before the experiment the dose rate was verified by the standard Fricke dosimeter. Three dosimeters were irradiated for each point of measurement.

\subsection{Basic of ESR Technique}

The direct detection of paramagnetic species consisting of one or more unpaired electrons in complex samples is ensured by Electron Spin Resonance (ESR) which is the standard spectroscopic technique. The basic principles of ESR consist on the interaction of electromagnetic radiation with magnetic moments caused by electrons (ESR). The magnetic moment of an unpaired electron arises from its "spin" and when placed within an external magnetic field, the electron spin will align parallel or antiparallel in the direction of the magnetic field, which corresponds to a lower $(\mathrm{Ms}=-1 / 2)$ or an upper $(\mathrm{Ms}=1 / 2)$ energy state. If electromagnetic radiation corresponding to the energy difference applied to the sample, resonance transition is possible between the lower and the upper energy states [19]. The energy difference $(\Delta \mathrm{E})$ between these two states is proportional to the strength of the applied magnetic field $\left(\mathrm{B}_{0}\right)$ :

$$
\Delta \mathrm{E}=\mathrm{h} v=\mathrm{g} \mu_{\mathrm{B}} \mathrm{B}_{0}
$$

where $\mathrm{h}$ is Planck's constant, $v$ is the frequency of the electromagnetic radiation, $g$ is a constant termed $g$ factor ( $\mathrm{g}=2.0023$ for an unpaired electron), and $\mu_{\mathrm{B}}$ is the Bohr magneton.

The commonly used ESR frequency in Standard Reference Dosimetry application is in the microwave range at $10 \mathrm{GHz}$ (X-band).

Table 1. Physical and chemical proprieties of PGA.

\begin{tabular}{|c|c|c|c|c|}
\hline Polymer & Melting Point $\left({ }^{\circ} \mathrm{C}\right)$ & Glass Transition $\left({ }^{\circ} \mathrm{C}\right)$ & Approximate Strength & Processing Method \\
\hline Polyesters: Poly (Glycolic Acid) & $225-230$ & $35-40$ & 7.0 GPa (Modulus) & $\mathrm{E}, \mathrm{IM}, \mathrm{CM}, \mathrm{SC}$ \\
\hline
\end{tabular}

$\mathrm{E}=$ extrusion; $\mathrm{IM}=$ injection moulding; $\mathrm{CM}=$ compression moulding; $\mathrm{SC}=$ solvent casting . 


\subsection{ESR Measurement}

EPR measurements at $9.837 \mathrm{GHz}$ (X-band) were performed at $25^{\circ} \mathrm{C}$ using an EMX Bruker spectrometer. The microwave power used, $316 \mathrm{~mW}$, were determined by considering the saturation properties of PGA EPR lines at $25^{\circ} \mathrm{C}$. The Sample were accommodated in quartz sample tubes and kept up from the bottom. The different EPR spectra presented in this paper have been normalized to the same receiver gain $\left(10^{3}\right)$ and sample weight $(\mathrm{mg})$ in order to get quantitative comparison of the EPR lines intensities between the different samples. All dosimeters were fixed in the cavity center and flat ones were oriented to lie in the cavity nodal plane. In the present work, it is thus very difficult to compare theses spectra to determine the absolute number of radical spins. Then, in this study, we will only use arbitrary units (arb.u.) to analyze the evolution of Radical concentration in PGA Polymer content as a function of the integrated dose.

\subsection{Determination of Keys ESR Parameters}

The PGA dosimeter was fixed in the cavity and measured 30 min after switching on the ESR spectrometer. Every spectrum was recorded in about $61 \mathrm{~s}$ and delay time was $300 \mathrm{~s}$. Microwave power, modulation amplitude, time constant, conversion time and modulation frequency were also studied, but are not shown here in detail. The operating EPR parameters were set as following: center magnetic field $351.5 \mathrm{mT}$, sweep width $5 \mathrm{mT}$, microwave power $316 \mathrm{~mW}$, modulation amplitude $1 \mathrm{G}$, modulation frequency $100 \mathrm{kHz}$, time constant $163.84 \mathrm{~ms}$ and conversion time $60 \mathrm{~ms}$.

\section{Results}

\subsection{ESR Spectra of $\boldsymbol{\gamma}$-Irradiated PGA}

Before gamma-irradiation, the PGA used in this study has small signal at about $348 \mathrm{mT}$ which can be related to impurity in PGA matrix. The X-Band ESR spectra were subsequently recorded at R.T. (Room Temperature, $25^{\circ} \mathrm{C}$ ). The resulting ESR spectrum of PGA sample after exposure to $25 \mathrm{kGy}$ is shown in Figure 2. All PGA samples, regardless of radiation dose produced similar EPR spectra varying only in the intensity of the observed signals.

The radiolytic degradation mechanism of PGA and it's copolymers due to irradiation of samples has been investigated previously $[12,20-22]$ and it's well known that chain scission processes dominate at low temperature. This results in a wide range of relative primary and secondary radical species which are visible by ESR. However at elevated temperatures (R.T.), as in ambient conditions studied here, Hydrogen abstraction reaction by the primary radicals occurs extensively at secondary $\mathrm{C}-\mathrm{H}$ bonds on the polymer backbone. This produces a nar- rower range of relatively stable radicals, such as $-\mathrm{CH}^{\circ}$ COO- which is visible at ambient temperature $\left(25^{\circ} \mathrm{C}\right)$ [12].

\subsection{Dose Response Curve}

In order to find out the useful dose range for this Polymer, the response curve (specific higher Peak to Peak (Hpp) versus dose) was measured in the dose range $0.1-200$ kGy (Figure 3). All data for the dose response curve were immediately acquired after irradiation. As expected in (Figure 3) the radical concentration increase linearly as function applied dose in the dose range $0.1-200 \mathrm{kGy}$ with the linear regression coefficient better than 0.99 (Figure 3). At higher doses the specific Hpp continued to grow slowly up to $200 \mathrm{kGy}$ which was the upper dose level of the present experiments. The PGA response had not yet reached saturation at this dose level. There was linear growth of the Hpp as function of the dose because at room temperature only one radical contribute to the ESR spectrum which is formed by hydrogen abstraction (Figure 1) RI - $\mathrm{CH}^{\circ}$-COO- [12]. In the dose range 0.1 to $10 \mathrm{kGy}$ there was also linear growth of the Hpp as function of the dose see (Inset Curve).

\subsection{Post-Irradiation Stability}

As a Reference Standard Dosimetry system, the response of PGA/ESR dosimetry system should have a good stability after irradiation because in addition of its use in calibration of radiation environment and routine dosime-

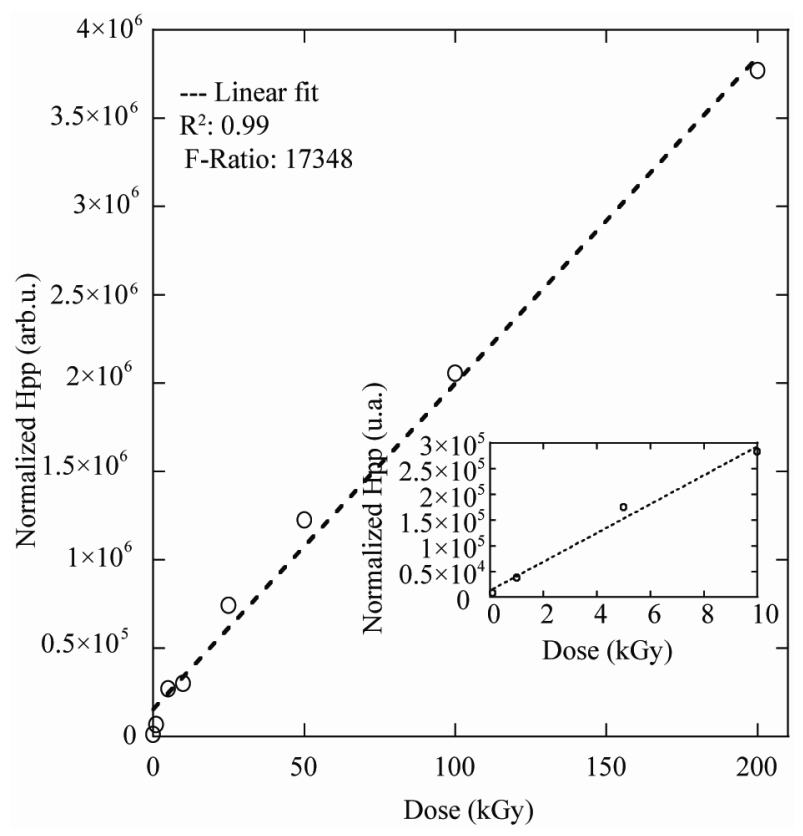

Figure 3. Dose response curve of PGA dosimeter in the dose range of 0.1 - $200 \mathrm{kGy}$, experimental ( $\left(^{\circ}\right)$ and calculated by a linear function (dashed line). 
ters it can be used as a Transfer Standard Dosimeter for transferring dose information from an accredited or national standards laboratory to an irradiation facility in order to establish traceability for that calibration facility. Hence the time stability of the radiation-induced radical is an important aspect in radiation dose measurement by ESR spectroscopy.

In our work the main difficulty presented by PGA dosimeter is the undesirable strong initial fading. In order to solve these problem PGA dosimeters were submitted to different post-irradiation thermal treatments from $60^{\circ} \mathrm{C}$ up to $140^{\circ} \mathrm{C}$ and the effect of these treatments on the fading behaviour of the dosimeters was studied up to 45 days after irradiation at room temperature.

\subsubsection{Radical Concentration Fading at Room Temperature}

In order to examine the stability of radical, three replicate samples PGA were irradiated with ${ }^{60} \mathrm{Co}$ gamma rays to $25 \mathrm{kGy}$. After irradiation PGA samples were stored at room Conditions: $25^{\circ} \mathrm{C}, 40 \%$ - $60 \% \mathrm{RH}$ (Relative $\mathrm{Hu}-$ midity). The resulting radical concentration was then followed up to 60 days via ESR technique.

The result concerning long-term room temperature variation in signal intensity was given in Figure 4. The signal intensity was found to be non stable at room temperature in the range of storage time. A function was used to fit signal intensity decay as shown in Figure 4. The coefficient of correlation $\left(\mathrm{R}^{2}\right)$ was 0.999 . The stability of radiation-induced radical at room temperature was mainly controlled by the fading process. This fading process seems to follow a simple first-order kinetic.

$$
\mathrm{Hpp}=110829,999+352107,206 \mathrm{e}^{(-0.096 \mathrm{x})}
$$

where $\mathrm{k}(0.096)$ is the first order decay rate constant.

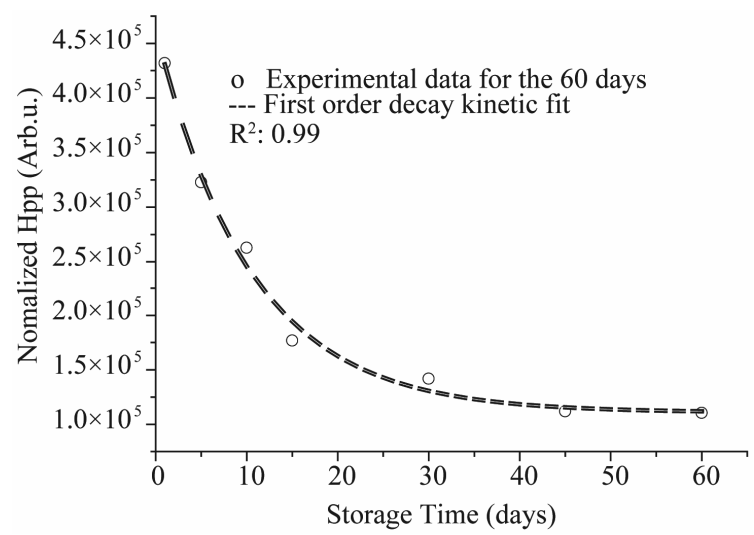

Figure 4. Experimental and calculated ESR signal intensity decay curves for PGA irradiated at a dose of $25 \mathrm{kGy}$ and kept at room temperature. Symbol (experimental) and dashed line (theoretical, calculated by a function describing firstorder kinetic).

\subsubsection{Effect of Post-Irradiation Heat Treatments on Radical Concentration Fading}

To get more insight in to the decay process of the free radical species, the effect of post-irradiation heat treatments on the PGA response fading was investigated. Six PGA samples were irradiated to a dose of $25 \mathrm{kGy}$ and immediately annealed at six different temperatures (R.T., $60^{\circ} \mathrm{C}, 80^{\circ} \mathrm{C}, 100^{\circ} \mathrm{C}, 120^{\circ} \mathrm{C}$ and $140^{\circ} \mathrm{C}$ ) for predetermined times $(35 \mathrm{~min})$, which was found to be the best heat treatment time. All ESR spectra are recorded at room temperature after cooling the samples to room temperature for predetermined times $(1,2,5,10,15,30,45$ days).

The radical concentration values were normalized to the first measurements taken $5 \mathrm{~min}$ after the heat treatments. The results are presented in Figure 5.

For each storage temperature, first-order kinetics model is used to fit the fading of the experimental data. The results show a remarkably difference between fading behaviour of PGA samples treated at various thermal treatments. Theoretical decreases in signal intensity calculated using the rate constant values determined after the fitting procedure were also given in Figure 5 (dashed lines) with their experimental counterparts. As it can be seen from this figure, the decreasing calculated and experimental decay data agree fairly well.

Post-irradiation heat treatments led to fast fading by accelerating the recombination of defects and the impurities diffusing into the PGA matrix. As it is expected (Figure 5), the lower temperature at the same annealing time correspond to the faster decays of the signal intensity.

The standard deviation (std) of PGA dosimeters response measurements is about $0.5 \%(1 \sigma)$ within the first day after irradiation and heating at $140^{\circ} \mathrm{C}$ for $35 \mathrm{~min}$ and about $3 \%-4 \%$ between the first and the 45 th days. Ac-

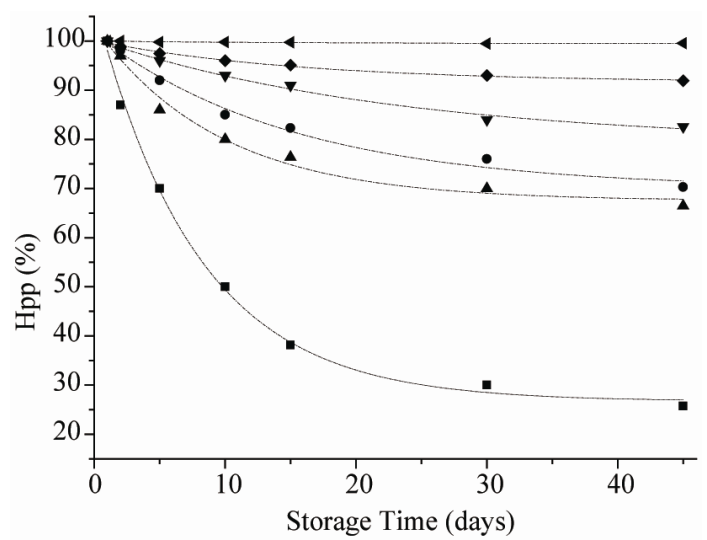

Figure 5. Variations of the ESR signal intensity with storage time for PGA samples irradiated at a dose of $25 \mathrm{kGy}$ and kept at different temperatures: $\mathrm{RT}(\square), 60^{\circ} \mathrm{C}(\Delta), 80^{\circ} \mathrm{C}(\bullet)$, $100^{\circ} \mathrm{C}(\nabla), 120^{\circ} \mathrm{C}(\diamond), 140^{\circ} \mathrm{C}(\varangle)$; calculated by a function describing first-order kinetic (dashed lines). 
cording to obtained results, the best results have been obtained with heat treatments at $140^{\circ} \mathrm{C}(35 \mathrm{~min})$.

This procedure is very effective for the removal of unstable entities responsible for the strong fading and it did not affect the metrological properties (reproducibility and useful dose range) of the PGA dosimeter.

The decay behaviours at the higher temperatures have been attributed to the semicrystalline nature of PGA, which will influence the mobility of the polymer chains, particularly in the crystalline and crystalline amorphous boundary regions [12]. The fact that most of the radicals formed in PGA decay well below the melting temperature of the crystallites suggests that they are formed in the amorphous regions of the polymer.

\subsubsection{First-Order Kinetic Behaviour of PGA Dosimeter}

Signal intensity calculated by second integral of any ESR spectrum is related to the radical populations in the sample and decrease at high temperatures due to the radical recombination transform to non-paramagnetic units. In this case, studying the variation of signal intensity above room temperature would be interesting from kinetic point of view of the free radical species in gamma-irradiated PGA.

Clearly, in Figure 5, it can be seen that radiation induced radical concentration (Hpp) is decreasing with time. The curve is much steeper at the beginning and as time progresses; the curve approaches a horizontal line. Thus, this graph evidently shows that the rate is dependent on how much radical concentration is left. How much does the rate depend on the species concentration? To answer this question, we need to find a linear plot and work with the integrated form of the rate law:

$$
\ln [\mathrm{Hpp}]=\ln \left(\mathrm{Hpp}_{0}\right)-\mathrm{kt}
$$

In the above equations $\left(\mathrm{Hpp}_{0}\right)$ is the initial concentration, $(\mathrm{Hpp})$ equals the concentration at time $(\mathrm{t})$ and $(\mathrm{k})$ is the first order constant.

Plotting $\ln [\mathrm{Hpp}]$ against time creates a linear plot up to a conversion of about $90 \%$ with slope $-\mathrm{k}$. As expected from Figure 6, a linear relationship is achieved between the natural log of radiation induced radical concentration (Hpp) and storage time (t) for each annealing temperature. According to the plot of $\ln [\mathrm{A}]$ versus time, one can see that the induced radical concentration (Hpp) fading process, seems to follow a simple first-order kinetic.

\subsubsection{Fading Constant Rate Evaluation}

The first-order kinetics fading rate constants $(k)$ were calculated for each storage temperature to fit the strong fading of the experimental data. The results show that the fading behaviour of the PGA samples is remarkably different for the various thermal treatments.
The observations indicate that the post irradiation heating $\left(140^{\circ} \mathrm{C}\right.$ for $\left.35 \mathrm{~min}\right)$ of the PGA samples correspond to the lower constant rate $(\mathrm{k})$ (Table 2), witch can traduced by a reducing in the radiation-induced radical concentration (Hpp), and an acceleration in the recombination of the entities responsible for the strong fading (Figure 5).

Consequently, the most suitable heating is $\left(140^{\circ} \mathrm{C}\right.$ for $35 \mathrm{~min}$ ); it can provide a good stability in radiation-induced radical concentration $(3 \%-4 \%$ for $1 \sigma)$ between the first and the 45th days. The evaluation of the PGA dosimeter can be performed immediately after irradiation and heat treatment.

\subsection{Effect of Post-Irradiation Heat Treatments on Radical Concentration}

In order to establish the useful temperature range of heat treatment and to preserve a detectable radiation induced radical response, the radical concentration was plotted against annealing temperature.

As exposed in Figure 7. The percentage loss in the to-

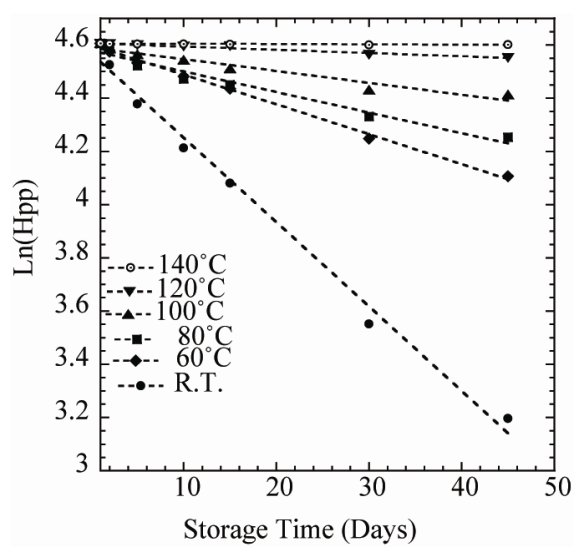

Figure 6. Variations of the natural logarithm of ESR signal intensity (Hpp) with storage time for PGA samples irradiated at a dose of $25 \mathrm{kGy}$ and heat treated for $35 \mathrm{~min}$ at different temperatures: RT (•), $60^{\circ} \mathrm{C}(\diamond), 80^{\circ} \mathrm{C}(\bullet), 100^{\circ} \mathrm{C}$ $(\Delta), 120^{\circ} \mathrm{C}(\nabla), 140^{\circ} \mathrm{C}(\circ)$.

Table 2. Calculated decay constants value for the proposed radical species contributing to the formation of ESR spectrum of gamma irradiated PGA for temperature range from $25^{\circ} \mathrm{C}$ to $140^{\circ} \mathrm{C}$.

\begin{tabular}{cc}
\hline Annealing Temperature $\left({ }^{\circ} \mathrm{C}\right)$ & Decay Constant $(\mathrm{k}) \times 10^{-2}\left(\right.$ day $\left.^{-1}\right)$ \\
\hline R.T. & 9.56 \\
60 & 6.17 \\
80 & 3.70 \\
100 & 3.35 \\
120 & 1.80 \\
140 & 1.35 \\
\hline
\end{tabular}


tal concentration of radicals on annealing to a temperature of $80^{\circ} \mathrm{C}$ was approximately $10 \%$, while annealing to $140^{\circ} \mathrm{C}$ caused a reduction of the radical concentration by approximately $70 \%$. The annealing experiments also revealed that $3 \%$ of the radicals remained at a temperature of $60^{\circ} \mathrm{C}$.

According to Figure 7 data, $140^{\circ} \mathrm{C}$ is a limit temperature which can not be exceeded caring the disappearance of irradiation induced radical ESR response and respecting the milting point at $225^{\circ} \mathrm{C}$, in which major modification in PGA structure may be uncounted.

\section{Conclusions}

A Poly Glycolic Acid (PGA) Polymer has been investigated with ESR technique in order to evaluate this potential as radiation-sensitive material for dose measurements. The response curve was measured in the dose range 0.1 kGy - 200 kGy. The results demonstrated that the influence of post-irradiation storage on the ESR/PGA response might be very significant. The heating at $140^{\circ} \mathrm{C}$ for 35 minutes was found to be the most suitable procedure to stabilize the response of irradiated PGA dosimeter.

The linearity of dose response curve and high stability of the radiation-induced radical species in $\gamma$-irradiated PGA, it was concluded that PGA presents wanted characteristics of a good dosimetric material and it has the potential to be used as a Reference and/or Transfer Standard Dosimetry System particularly for ${ }^{60} \mathrm{Co} \gamma$-ray.

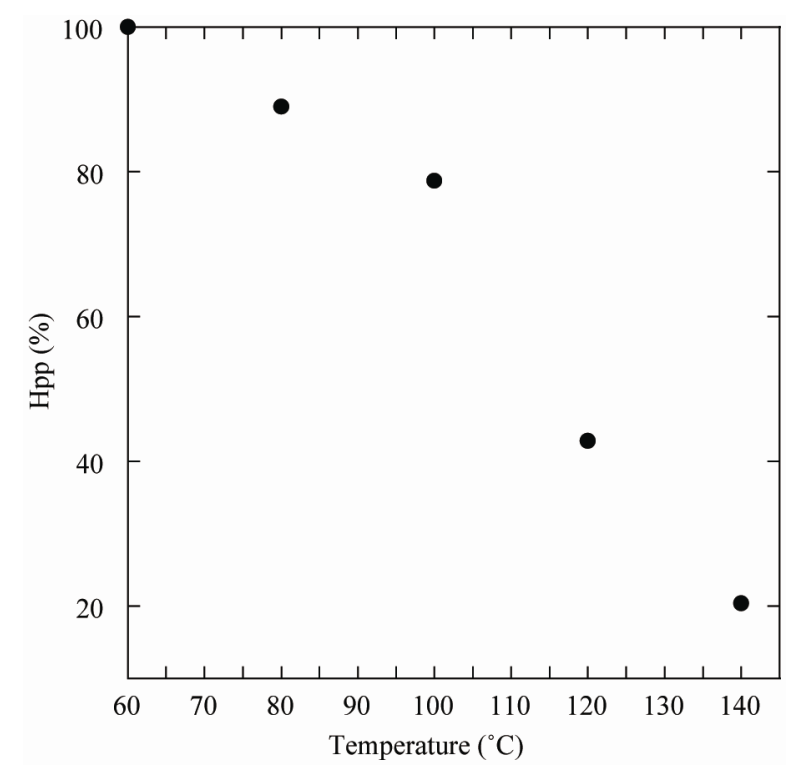

Figure 7. Percentage of radicals remaining in Gammairradiated Poly Glycolic Acid (PGA) after annealing at different temperatures for $35 \mathrm{~min}$, measurements were done immediately after irradiation and heating.

\section{REFERENCES}

[1] G. D. Watkins, "EPR of Defects in Semiconductors: Past, Present, Future," Physics of the Solid State, Vol. 41, No. 5, 1999, pp. 746-750. doi:10.1134/1.1130862

[2] A. L. Konkin, H. K. Roth, M. Schroedner, G. A. Nazmutdinova, A. V. Aganov, T. Ida and R. R. Garipov, "TimeResolved EPR Study of Radicals from 2,2-Dimethoxy2-Phenylacetophenone in Ethylene Glycol after Flash Photolysis," Chemical Physics, Vol. 287, No. 3, 2003, pp. 377-389.

[3] M. Bennati and T. F. Prisner, "New Developments in High Field Electron Paramagnetic Resonance with Applications in Structural Biology," Reports on Progress in Physics, Vol. 68, No. 2, 2005, p. 411. doi:10.1088/0034-4885/68/2/R05

[4] D. F. Regulla and U. Deffner, "Dosimetry by ESR Spectroscopy of Alanine," The International Journal of Applied Radiation and Isotopes, Vol. 33, No. 11, 1982, pp. 1101-1114.

[5] M. Ikeya, "New Applications of Electron Spin Resonance: Dating, Dosimetry, and Microscopy," World Scientific Publishing, Singapore, 1993.

[6] T. Kjima and R. Tnaka, "Polymer-Alanine Dosimeter and Compact Reader," International Journal of Radiation Applications and Instrumentation. Part A. Applied Radiation and Isotopes, Vol. 40, No. 10-12, 1989, pp. 851-857. doi:10.1016/0883-2889(89)90006-3

[7] S. P. Dias, A. M. Rossi, R. T. Lopes and E. F. O. de Jsus, "Evaluationof Dosimetric Properties of Paramagnetic Centers Formed in Gamma Irradiated Polymers," Radiation Protection Dosimetry, Vol. 85, No. 1-4, 1999, pp. 463-468.

[8] V. Gancheva, E. Sagstuen and N. D. Yordanov, "Study on the EPR/Dosimetric Properties of Some Substituted Alanines," Radiation Physics and Chemistry, Vol. 75, No. 2, 2006, pp. 329-335.

[9] G. M. Hassan and M. A. Sharaf, "EPR Dosimetric Properties of Some Biomineral Materials," Applied Radiation and Isotopes, Vol. 62, No. 2, 2005, pp. 375-381. doi:10.1016/j.apradiso.2004.08.013

[10] A. Lund, S. Olsson, M. Bonora, E. Lund and H. Gustafsson, "New Materials for ESR Dosimetry," Spectrochimica Acta Part A: Molecular and Biomolecular Spectroscopy, Vol. 58, No. 6, 2002, pp. 1301-1311. doi:10.1016/S1386-1425(01)00719-3

[11] E. Lund, H. Gustafsson, M. Danilczuk, M. D. Sastry, A. Lund, T. A. Vestad, E. Malinen, E. O. Hole and E. Sagstuen, "Formates and Dithionates: Sensitive EPRDosimeter Materials for Radiation Therapy," Applied Radiation and Isotopes, Vol. 62, No. 2, 2005, pp. 317-324. doi:10.1016/j.apradiso.2004.08.015

[12] V. Gancheva, E. Sagstuen and N. D. Yordanov, "Study on the EPR/Dosimetric Properties of Some Substituted Alanines," Radiation Physics and Chemistry,Vol. 75, No. 2, 2006, pp. 329-335. doi:10.1016/j.radphyschem.2005.03.009

[13] A. Babanalbandi, D. J. T. Hill, J. H. O'Donnell and P. J. Pomery, "An Electron Spin Resonance Analysis on Y- 
Irradiated Poly (Glycolic Acid) and Its Copolymers with Lactic Acid," Polymer Degradation and Stability, Vol. 52, No. 1, 1996, pp. 59-61. doi:10.1016/0141-3910(95)00230-8

[14] R. K. Kulkarni, K. C. Pani, C. Neuman and F. Leonard, "Polylactic Acid for Surgical Implants," US National Technical Information Service, AD Report No. 636716, 1966, pp. 1-17.

[15] D. E. Cutright, J. M. Brady, R. A. Miller and M. A. J. Willis, "Systemic Mercury Levels Caused by Inhaling Mist during High-Speed Amalgam Grinding," Journal of Oral Medicine, Vol. 28, No. 4, 1973, pp. 100-104.

[16] B. Engin, C. Aydas and H. Demirtas, "ESR Dosimetric Properties of Window Glass," Nuclear Instruments and Methods in Physics Research Section B: Beam Interactions with Materials and Atoms, Vol. 243, No. 1, 2006, pp. 149-155. doi:10.1016/j.nimb.2005.08.151

[17] A. de A. Rodrigues Jr. and L. V. E. Caldas, "Commercial Plate Window Glass Tested as a Routine Dosimeter at a Gamma Irradiation Facility," Radiation Physics and Chemistry, Vol. 63, No. 3-6, 2002, pp. 765-767. doi:10.1016/S0969-806X(01)00637-5

[18] K. Farah, T. Jerbi, F. Kuntz and A. Kovacs, "Dose Meas- urements for Characterization of a Semi-Industrial Cobalt-60 Gamma-Irradiation Facility," Radiation Measurements, Vol. 41, No. 2, 2006, pp. 201-208. doi:10.1016/j.radmeas.2005.03.003

[19] J. A. Weil and J. R. Bolton, "Electron Paramagnetic Resonance: Elementary Theory and Practical Applications," 2nd Edition, John Wiley \& Sons, Hoboken, 2007.

[20] A. G. Hausberger, R. A. Kenley and P. P. DeLuca, "Gamma Irradiation Effects on Molecular Weight and in Vitro Degradation of Poly (D, L-Lactide-Co-Glycolide) Microparticles," Pharmaceutical Research, Vol. 12, No. 6, 1995, pp. 851-856. doi:10.1023/A:1016256903322

[21] M. B. Sintzel, A. Merkli, C. Tabatabay and R. Gurney, "Influence of Irradiation Sterilization on Polymers Used as Drug Carriers-A Review," Drug Development and Industrial Pharmacy, Vol. 23, No. 9, 1997, pp. 857-879. doi: $10.3109 / 03639049709148693$

[22] L. Montanari, M. Constantani, E. Ciranni-Signoretti, L. Valvo, M. Santucci, M. Barolomei, P. Fattibene, S. Onori, A. Faucitano, B. Conti and I. Genta, "Gamma Irradiation Effects on Poly (DL-Lactide-Co-Glycolide) Microspheres," Journal of Controlled Release, Vol. 56, No. 1-3, 1998, pp. 219-229. doi:10.1016/S0168-3659(98)00082-0 